Relations industrielles

Industrial Relations

\title{
La représentation collective au travail en contexte d'externalisation des services publics d'aide à domicile au Québec
}

\author{
Collective Representation at Work in the Context of \\ Outsourcing of Public Homecare Services in Quebec \\ La representación colectiva laboral en contexto de \\ externalización de servicios públicos de asistencia a domicilio \\ en Quebec
}

\section{Louise Boivin}

Volume 72, numéro 3, été 2017

Les nouvelles frontières de la relation d'emploi

New Frontiers of the Employment Relationship

URI : https://id.erudit.org/iderudit/1041095ar

DOI : https://doi.org/10.7202/1041095ar

Aller au sommaire du numéro

Éditeur(s)

Département des relations industrielles de l’Université Laval

ISSN

0034-379X (imprimé)

1703-8138 (numérique)

Découvrir la revue

Citer cet article

Boivin, L. (2017). La représentation collective au travail en contexte d'externalisation des services publics d'aide à domicile au Québec. Relations industrielles / Industrial Relations, 72(3), 501-523.

https://doi.org/10.7202/1041095ar

\section{Résumé de l'article}

L'externalisation, qui donne lieu à l'intégration indirecte du travail dans l'organisation productive, pose des défis importants pour la représentation collective des travailleuses et des travailleurs. C'est que le droit du travail a été établi en fonction d'un tout autre modèle organisationnel. Afin de mieux comprendre ces défis, nous avons mené trois études de cas sur la représentation collective en contexte d'externalisation des services publics d'aide à domicile au Québec durant la période 2003-2013. Les travailleuses concernées - majoritairement des femmes - occupent des emplois précaires chez trois types de prestataires privés intégrés à des réseaux locaux de services: entreprises d'économie sociale en aide domestique (EESAD), usagers du programme Chèque emploi-service (CES) et agences de location de personnel. Nous avons examiné si des pratiques de représentation collective de ces travailleuses existent et quels acteurs sociaux les portent. Nous avons aussi vérifié si ces pratiques se confinent à l'intérieur des frontières de l'entité identifiée comme l'employeur au sens juridique ou si elles sont " réticulaires ", étendant la solidarité à la sphère du pouvoir stratégique (Appay, 1997) exercé par les autorités publiques dans les réseaux.

Nos résultats montrent l'absence d'une représentation collective réticulaire dans ces réseaux locaux de services où la dévalorisation sexuée du travail, contrée en partie dans le secteur public, revient en force. Le personnel de 15\% des EESAD est syndiqué, mais les pratiques de représentation syndicale n'interpellent que l'employeur reconnu au sens juridique, les EESAD. Dans les agences de location de personnel intégrées à ces réseaux locaux, aucune forme de représentation collective n'existe, ni dans le programme CES. Cependant, une action collective interpellant les autorités publiques au sujet des conditions d'emploi dans le CES a eu un certain succès ponctuel. Portée par une coalition d'associations locales représentant des personnes vivant avec des limitations fonctionnelles, elle ouvre la voie à l'idée d'alliances salariées-usagers autour de la qualité des services et de l'emploi.
Tous droits réservés @ Département des relations industrielles de l’Université Laval, 2017
Ce document est protégé par la loi sur le droit d'auteur. L’utilisation des services d'Érudit (y compris la reproduction) est assujettie à sa politique d'utilisation que vous pouvez consulter en ligne.

https://apropos.erudit.org/fr/usagers/politique-dutilisation/ 


\section{La représentation collective au travail en contexte d'externalisation des services publics d'aide à domicile au Québec}

\section{Louise Boivin}

L'externalisation pose des défis importants pour la représentation collective alors que le droit du travail y est peu adapté. Nous avons mené trois études de cas sur la situation de travailleuses précaires - majoritairement des femmes - employées par des prestataires privés qui sont intégrés aux réseaux locaux de services d'aide à domicile au Québec. Nos résultats montrent l'absence d'une "représentation collective réticulaire " portée par les syndicats, c'est-à-dire une représentation s'étendant à la sphère du " pouvoir stratégique " (Appay, 1997) exercé par les autorités publiques, entités non reconnues comme employeur au sens juridique. Pourtant, ce pouvoir a un effet déterminant sur les conditions d'exercice du travail et sur sa dévalorisation sexuée. Seule une coalition d'associations locales de défense des droits des personnes vivant avec des limitations fonctionnelles, usagères des services, a interpellé les autorités publiques en vue d'une amélioration de la qualité des emplois et des services.

MOTS-CLÉs: réseau, syndicalisme, relations de travail triangulaires, précarité, privatisation.

\section{Introduction}

L'externalisation ${ }^{1}$, par le recours à des moyens tels la sous-traitance, le travail autonome ou les agences de location de personnel, est au cœur des pratiques de gestion des entreprises et des organismes publics à la recherche de flexibilité et d'une réduction des coûts de production et de main-d'œuvre. Elle soulève des enjeux importants pour la représentation collective des travailleuses et des

Louise Boivin, professeure, département de relations industrielles, Université du Québec en Outaouais (UQO), Gatineau, Québec (Louise.Boivin@uqo.ca).

L'auteure souhaite remercier les personnes qui ont dirigé et édité ce numéro spécial de la revue, de même que celles qui ont évalué l'article pour leurs judicieux commentaires. Elle remercie également Guy Bellemare pour ses commentaires sur une version antérieure de ce texte. De même, elle remercie Guylaine Vallée, professeure titulaire à l'École de relations industrielles de l'Université de Montréal, qui a supervisé la thèse de doctorat de laquelle est tirée la majeure partie des résultats présentés dans l'article. Enfin, elle remercie le Conseil de recherches en sciences humaines (CRSH) et le Centre de recherche interuniversitaire sur la mondialisation et le travail (CRIMT) pour leur soutien financier à cette recherche. 
travailleurs $^{2}$, et ce, même lorsqu'elle s'inscrit dans une seule juridiction. Les nouvelles configurations organisationnelles sous forme de réseau ${ }^{3}$ (Boltanski et Chiapello, 1999) qui résultent de l'externalisation tranchent avec le modèle de l'entreprise verticalement intégrée de type fordiste et de la relation de travail bipartite employeur-salarié. C'est en fonction de ce modèle que se sont forgés le droit du travail, ainsi que les structures et pratiques du mouvement syndical dans les pays industrialisés (Gumbrell-McCormick et Hyman, 2013). Avec l'externalisation, l'intégration du travail dans l'organisation productive s'opère de façon indirecte "par le recours à un amalgame de contrats qui fait en sorte que l'entrepreneur bénéficie du travail de quelqu'un sans être considéré comme son employeur, ce que l'on peut qualifier de stratégie "d'externalisation" des responsabilités de l'employeur » (Vallée, 2005: 7). La difficile application du droit du travail à ces situations de travail indirect (Davidov, 2015) et à ces relations de travail triangulaires, voire multipartites, vient considérablement affaiblir le filet de sécurité instauré par les États sociaux et engendre une précarisation de l'emploi (Bernstein et al., 2009).

Dans le présent article, la question qui nous intéresse est celle de savoir quelles sont les pratiques de représentation collective les plus susceptibles de contribuer à la construction d'un pouvoir collectif pour les travailleuses et les travailleurs se retrouvant dans des relations de travail multipartites générées par l'externalisation. Nous concevons ce pouvoir collectif, non seulement dans une perspective d'amélioration de la rémunération et des conditions de travail, mais aussi dans celle d'un accroissement de la capacité d'action sociale-politique, ce qui constitue une expression de la liberté (Pinard, 2000). Pour traiter de cette question, nous avons étudié l'externalisation des services publics ${ }^{4}$ d'aide à domicile au Québec vers trois types de prestataires privés, soit les entreprises d'économie sociale (EESAD), les usagers du programme du Chèque emploi-service (CES) et les agences de location de personnel. Nous analysons spécifiquement la représentation des travailleuses ${ }^{5}$ employées par ces prestataires privés dans le cadre de trois études de cas durant la période 2003-2013. Ce secteur des services $d^{\prime}$ aide à domicile, en pleine expansion dans plusieurs pays avec le vieillissement de la population, est caractérisé par une forte précarisation de l'emploi et une faible valorisation sociale du travail (Weber, Trabut et Bellaud, 2014; Avril, 2009), ainsi que d'importantes difficultés de représentation collective (Béroud, 2013; Boris et Klein, 2006; Cranford, 2005). Dans les prochaines sections, nous exposerons notre cadre analytique, le cadre institutionnel des rapports collectifs au Québec, de même que notre méthodologie de recherche. Après avoir résumé les transformations organisationnelles dans lesquelles s'inscrit l'externalisation des services publics d'aide à domicile au Québec, nous présenterons nos résultats empiriques et, enfin, leur discussion. 


\section{Cadre analytique}

L'analyse des pratiques de représentation collective dans une perspective de construction du pouvoir collectif des travailleuses et des travailleurs employés au sein de relations de travail multipartites découlant de l'externalisation doit préalablement reposer, à notre avis, sur une conceptualisation des rapports de pouvoir dans un tel contexte organisationnel. Certains économistes néoinstitutionnalistes expliquent la dynamique de pouvoir et la régulation des réseaux selon le type de division de la production entre les entreprises (horizontale ou verticale) (Baudry, 2005; Moati, 2006). Pour leur part, des chercheurs en géographie sociale réfèrent à certains processus sociaux et institutionnels dans les réseaux de production locaux afin d'expliquer les disparités de pouvoir entre les entreprises qui les composent (Christopherson et Clark, 2007; Zeitlin, 2008). En sociologie des organisations, Mariotti (2005) s'est, pour sa part, intéressé à ce qu'il appelle la "capacité de gouverner» dans le réseau, ainsi qu'aux mécanismes de gouvernement. Malgré leur apport pour la compréhension des dynamiques économiques et sociales au sein des réseaux, ces travaux n'intègrent pas une analyse sociologique du travail et occultent donc sa centralité dans ces dynamiques. Les études de la sociologue du travail Béatrice Appay nous sont apparues fort pertinentes à cet égard.

S'inscrivant dans le courant théorique de l'économie politique critique et du procès de travail (Labour process, en anglais), elle nomme le mode productif postfordiste " autonomie contrôlée », conceptualisant celle-ci comme un processus contradictoire de centralisation sur le plan financier et d'externalisation basée sur la "sous-traitance en cascade» sur le plan organisationnel (Appay, 2005: 45). Un transfert des risques économiques et sociaux vers les salariés des sous-traitant en résulte: «Cette division du travail interentreprises, fortement polarisatrice, ne permet-elle pas de contourner les régulations salariales, celles en particulier ayant trait à la subordination entre salariés et employeurs, subordination qui est au fondement du droit du travail contemporain » (Appay, 1997: 540). L'externalisation productive participe d'un processus qui «fragmente, divise, hiérarchise, organise structurellement l'impuissance syndicale, désorganise les luttes au travail» (Appay, 2008: 166). Dans ce processus, ce qu'elle appelle les brain firms (Appay, 1998: 172), que nous traduisons par "organisations-cerveaux ${ }^{6}$, exercent au sein des réseaux un "pouvoir stratégique»(Appay, 1997). Ce dernier dépend de l'autonomie et de la coopération des diverses entités juridiques composant le réseau, qui se voient, par ailleurs, imposer des marges de manœuvre restreintes dans leur action (Appay, 2005: 77-78).

En recourant au cadre analytique d'Appay lors de nos travaux antérieurs portant sur les situations d'emploi dans les réseaux locaux d'aide à domicile au Québec, nous avons constaté que la protection des droits de représentation collective des 
travailleuses à l'emploi des prestataires privés intégrés à ces réseaux était peu effective (Boivin, 2013a; 2015). En effet, en vertu de la régulation juridique actuelle, l'employeur identifié est le prestataire privé, alors que ce sont les autorités publiques qui constituent les organisations-cerveaux exerçant le pouvoir stratégique dans les réseaux locaux, pouvoir ayant un effet déterminant sur les conditions d'exercice du travail de ces salariées. Ce pouvoir stratégique se manifeste sous quatre aspects: autonomie juridique formelle des entités composant le réseau; maitrise financière des principaux canaux financiers par le ministère de la Santé et des Services sociaux (MSSS); " orchestration » de la production des services par le MSSS et les Centres de santé et de services sociaux (CSSS) - des organismes publics locaux -; et «régulation supra-organisation» par les CSSS7. Dans ce contexte, les autorités publiques n'ont aucune obligation juridique à l'égard des salariées embauchées par des prestataires privés, des travailleuses qui se retrouvent dans des emplois fortement précarisés où la dévalorisation sexuée du travail est renforcée, comparativement à la situation des employées du secteur public.

Pour analyser les pratiques de représentation collective dans le cadre de l'externalisation des mêmes services publics et des organisations en réseau qui en résultent, nous reprenons, cette fois, le concept de "pouvoir stratégique» d'Appay, complété par des éléments tirés des travaux d'Altmann et Dei $\beta$ (1998) sur la construction du pouvoir collectif des salariés. Même si les travaux de ces derniers portent sur les réseaux dans le secteur manufacturier en Allemagne, les constats qui en ressortent nous apparaissent adaptables aux services d'aide à domicile. Comme Appay, ces auteurs relèvent que la fragmentation de la main-d'œuvre au sein des réseaux entraine un affaiblissement de son pouvoir collectif, car les intérêts divergents et la compétition entre segments des réseaux viennent miner la solidarité. Ils constatent la nécessité d'une représentation collective organisée sur toute la chaîne, basée sur la solidarité entre la main-d'œuvre des différents maillons (Altmann et Dei $\beta, 1998:$ 151). Ces chercheurs concluent que la légitimité des syndicats comme force politique dans la société est liée à leur capacité d'exprimer une vision dépassant la segmentation organisée entre les salariés de différentes entreprises composant une même chaîne de production (ibid. : 152).

Compte tenu des similitudes avec nos études de cas, c'est donc sur ce cadre théorique que nous basons notre analyse des pratiques de représentation collective des travailleuses employées par les prestataires privés dans les réseaux locaux de services. Nous examinerons si ces pratiques traduisent une prise en compte du pouvoir stratégique exercé dans les réseaux et si elles se basent sur une construction de la solidarité dépassant les divisions instaurées entre segments des réseaux, ce que nous avons conceptualisé comme des pratiques de «représentation collective réticulaire». Par ailleurs, comme les syndicats ne sont pas les seuls acteurs pouvant potentiellement exercer ces pratiques, nous 
examinerons aussi si de « nouveaux acteurs » les portent, en reprenant la définition de Bellemare selon laquelle ceux-ci doivent avoir «la capacité d'influencer directement l'orientation des relations industrielles, y compris celle d'influencer les capacités causales déployées par d'autres acteurs des relations industrielles (action indirecte)»(Bellemare, 2000: 386, notre traduction).

\section{Cadre institutionnel et méthodologie de la recherche}

Les services d'aide à domicile au Québec sont dispensés auprès de personnes âgées, vivant avec des limitations fonctionnelles ou en convalescence. Selon la définition officielle du ministère, ils intègrent les composantes suivantes: services d'assistance à la personne (soins d'hygiène, assistance pour les déplacements et I'alimentation, etc.), aussi appelés Activités de la vie quotidienne (AVQ); services d'aide domestique (entretien ménager, préparation des repas, etc.), aussi appelés Activités de la vie domestique (AVD); et, enfin, activités de soutien civique (administration du budget, formalités administratives, etc.) (MSSS, 2004: 41).

Le régime général de rapports collectifs québécois s'applique aux travailleuses employées par des prestataires privés pour dispenser ces services, alors que celles du secteur public sont couvertes par le régime spécifique de ce secteur. Le régime général est décentralisé, s'inspirant fortement du modèle étatsunien du Wagner Act. Le syndicat qui obtient l'appui d'une majorité d'un groupe donné de salariés se voit attribuer un monopole de représentation pour l'ensemble de ces salariés dans cette unité d'accréditation. Ce groupe peut être constitué de tous les salariés d'un même employeur ou, le plus souvent, des salariés œuvrant dans un seul établissement de celui-ci. Précisons que le régime général ne permet pas l'accréditation multipatronale.

Les résultats de l'analyse qualitative présentée dans cet article proviennent de nos trois études de cas. Les données, recueillies entre 2011 et 2013, ont été triangulées. Le premier type de source de données, utilisé uniquement pour les deux premiers cas (économie sociale et chèque emploi-service), repose sur une enquête terrain dans deux réseaux locaux de régions différentes, comprenant une quinzaine d'entrevues avec les acteurs impliqués (gestionnaires publics et privés, deux travailleuses par réseau, personnes représentant des organisations syndicales et une association locale). Le contenu de conventions collectives et de documents produits par les acteurs sociaux impliqués dans ces réseaux a aussi été analysé. Le second type de source est constitué du contenu de quinze conventions collectives s'appliquant dans les EESAD et de la convention du secteur public, ainsi que le contenu des documents produits par les autorités publiques, des organisations syndicales nationales et des associations. Soulignons qu'afin de protéger l'anonymat des individus et des organisations qui ont collaboré à notre recherche, aucun ne sera identifié dans ce texte. 


\section{Les transformations de l'organisation des services d'aide à domicile ${ }^{8}$}

Les services publics d'aide à domicile s'inscrivent dans la catégorie des services sociaux et de santé, domaine qui est de juridiction provinciale au Canada. Ces services, mis en place en 1979 au Québec dans le sillage de la première politique de soutien à domicile, visaient à répondre à des besoins croissants liés au vieillissement de la population et à la diminution de l'hébergement des personnes âgées en institution, en vue de leur maintien le plus longtemps possible dans leur milieu de vie. Les services étaient alors dispensés par les Centres locaux de santé et de services communautaires (CLSC), des organismes publics créés à travers tout le Québec. Les travailleuses dispensant ces services, les auxiliaires familiales et sociales ${ }^{9}$, ont vu leur qualification professionnelle reconnue avec la mise en place d'un programme de formation d'environ 975 heures, ce qui a permis de rompre avec l'idée de «vocation féminine» associée à leur travail (Bourque, 1991). Cette reconnaissance a aussi résulté de leur syndicalisation et de la négociation collective de leurs salaires et de leurs conditions de travail dans le cadre du régime centralisé de rapports collectifs spécifique au secteur public québécois. Les tâches de ces auxiliaires ont été enrichies grâce à leur intégration dans des équipes multidisciplinaires de personnel professionnel (Bélanger et al., 1987). L'étatisation des services et la reconnaissance de la qualification professionnelle exigée par le travail ont également été favorisées par la mobilisation du mouvement féministe de la deuxième vague qui revendiquait une prise en charge par l'État de ces services et qui souhaitait une valorisation de certaines fonctions dévolues aux femmes dans la sphère domestique (Lamoureux, 1998).

Dès les années 1980, et de façon plus intense à partir de la moitié des années 1990, les gouvernements successifs ont réduit les services dispensés dans les hôpitaux et les centres d'hébergement publics. De même, une partie de la production des services publics d'aide à domicile a été externalisée vers des prestataires privés. À partir de la fin 2003, l'externalisation s'est amplifiée avec la réforme lancée par le gouvernement libéral nouvellement élu (MSSS, 2003). Le ministère de la Santé et des Services sociaux (MSSS) structure alors les services sociaux et de santé autour de 95 réseaux locaux répartis sur le territoire québécois, chacun coordonné par des organismes publics locaux, les Centres de santé et de services sociaux (CSSS). Ces derniers découlent de la fusion des institutions publiques de chaque territoire, dont les CLSC. Dans le cadre de ces réseaux, les services d'aide à domicile sont dispensés par les CSSS, ainsi que par le personnel employé par trois types de prestataires privés. Nous les présentons dans les trois encadrés qui suivent: 1- les entreprises d'économie sociale en aide domestique (EESAD); 2-les usagers recourant au programme gouvernemental du Chèque emploi-service (CES); et 3- les agences de location de personnel. 


\section{Encadré 1}

\section{Les entreprises d'économie sociale en aide domestique (EESAD)}

Les EESAD peuvent être définies comme des organismes dont l'activité principale est de produire des biens et services en poursuivant des objectifs sociaux. Leur statut juridique est celui d'organisme sans but lucratif ou de coopérative. En 2011, 101 EESAD étaient accréditées au Québec. En 2008-2009, un total de 6520 personnes occupaient un emploi dans les EESAD (emploi administratif ou de terrain), un total parmi lesquelles $46 \%$ travaillaient à temps partiel. Les femmes constituaient $93 \%$ de ce personnel. La classe d'âge la plus représentée dans les emplois est celle des 46-55 ans (MAMROT, 2012 : 21). Les horaires éclatés sur la journée ou la semaine, variables et imprévisibles, sont courants pour ce personnel (Boivin, 2013a). En 2011, le taux salarial horaire moyen des travailleuses à l'emploi d'EESAD syndiquées dispensant des services d'assistance à la personne était de 11,29\$ (ibid.: 361), soit $64 \%$ du taux salarial horaire du personnel du secteur public au premier échelon de l'échelle salariale effectuant des tâches similaires (MSSS, 2010). II est à noter que ce calcul exclut l'écart important sur le plan des avantages sociaux (assurances collectives, régime de retraite, congés, etc.).

\section{Encadré 2}

\section{Le programme du Chèque emploi-service (CES)}

Le CES constitue une variante des programmes existant dans divers pays ( $\mathrm{Da}$ Roit et Le Bihan, 2010) et nommés en anglais Consumer-directed ou Cash-for-care Programs. II prend la forme d'une allocation financière attribuée par le CSSS pour un nombre précis d'heures de services à l'usagère ou l'usager qui embauche la personne de son choix, à l'exception d'un membre de sa famille. En 2012, 10036 personnes travaillaient dans le cadre de ce programme, dont $94 \%$ de femmes*. Aucune donnée officielle n'est disponible sur la proportion de personnel employé à temps partiel ni sur l'âge moyen des travailleuses. $78 \%$ des services étaient dispensés à des personnes handicapées et $18 \%$ à des personnes âgées (MSSS, 2011a). En 2011, le taux salarial horaire moyen $(11,37 \$$ ) représentait $64 \%$ du taux salarial horaire du personnel du secteur public au premier échelon de l'échelle salariale effectuant des tâches similaires (MSSS, 2010), sans compter l'écart sur le plan des avantages sociaux. De plus, les frais d'essence et d'entretien de la voiture personnelle utilisée pour les déplacements ne sont pas remboursés. Les horaires à temps partiel, éclatés, variables et imprévisibles sont également courants dans le CES (Boivin, 2013a).

* Données provenant d'un courriel de la Direction du soutien à domicile du MSSS daté du 7 mars 2016, en complément d'un document remis à la suite de notre demande d'accès à l'information du 11 novembre 2015. 


\section{Encadré 3}

\section{Les agences de location de personnel}

Spécifions d'abord que la régulation des activités des agences est très faible au Québec et que le marchandage n'y est pas interdit, c'est-à-dire qu'il est légal pour une entreprise ou une administration publique de recourir à une agence dans le but de combler des besoins permanents de personnel (Bernier, Vallée et Jobin, 2003 : 496). Les agences privées dans les services d'aide à domicile sont, en général, des entreprises locales appartenant à d'anciens professionnels de la santé devenues entrepreneurs ou à des filiales de multinationales. Les agences locales emploient généralement une dizaine de personnes à temps plein et environ 200 personnes sur appel, alors que les filiales peuvent employer jusqu'à 500 personnes (Meintel, Fortin et Cognet, 2006: 565). II n'existe pas de données publiques sur les salaires, le statut d'emploi, le sexe, et l'âge du personnel des agences intégrées aux réseaux locaux. Des données officielles indiquent, cependant, que le montant consacré par les établissements publics à l'achat de services d'aide à domicile auprès d'agences de location de personnel a augmenté de $84 \%$ entre les années fiscales 2005-2006 et 2009-2010 dans la région de Montréal, où se trouve la plus forte concentration d'agences (MSSS, 2011b*: 195-207). Le travail sur appel et à temps partiel, basé sur des horaires éclatés, variables et imprévisibles, est courant dans ces agences (Boivin, 2013a; Meintel, Fortin et Cognet, 2006).

* Le document duquel nous avons tiré ces données réfère aux services dispensés dans le secteur public par la catégorie de personnel identifiée comme étant les « auxiliaires familiales ». Le montant investi pour l'achat de services auprès d'agences de location de personnel dans cette catégorie est passé de 11977651 \& en 2005-2006 à 22037360 \$ en 2009-2010 pour la région de Montréal (excluant la région de Laval).

À la suite de la réorganisation des services lancée en 2003, les CSSS ont la responsabilité de coordonner les réseaux locaux. Les auxiliaires familiales et sociales à leur emploi dispensent les services d'assistance à la personne de courte durée (moins de trois mois). Ces services s'adressent à des personnes dont l'état est instable ou nécessitant des soins postopératoires. Pour leur part, les travailleuses des EESAD et celles du CES dispensent les services d'assistance personnelle requis sur une longue durée (plus de trois mois) (MSSS, 2004). Ces services peuvent, sous certaines conditions ${ }^{10}$, inclure des actes de type médical, réservés au personnel infirmier jusqu'en 2002. Bien que la politique ministérielle ne le précise pas, les données officielles (voir Encadré 3) révèlent que le personnel des agences de location de personnel intégrées aux réseaux locaux dispense aussi des services d'assistance à la personne, dont des services de longue durée (MSSS, 2011b).

Certaines données récentes, qui n'étaient pas disponibles au moment où nous avons réalisé notre collecte de données pour la période 2003-2013, montrent que cette externalisation des services de longue durée prévue dans la réforme de 2003-2004 s'est concrètement réalisée. La Figure 1 ci-dessous présente, pour 
I'année fiscale 2014-2015, la répartition des heures de services d'aide à domicile longue durée sous l'égide des CSSS selon le type de prestataire. L'on y constate que la part des heures de services de longue durée dispensée par les travailleuses du CES est la plus élevée, soit $43 \%$, alors que celle dispensée par le personnel des CSSS représente $27 \%$, tandis que celle dispensée conjointement par les EESAD et les agences de travail temporaire représente 30\% (les données disponibles n'établissement pas de distinction entre les deux).

FIGURE 1

Heures de services d'aide à domicile longue durée selon le type de prestataire en 2014-2015

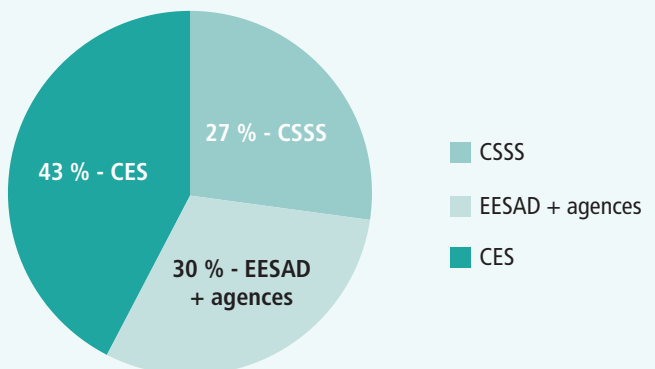

Données compilées par l'auteure à partir du tableau « Nombre d'heures travaillées en soutien à domicile longue durée» produit par la Direction générale adjointe des services aux aînés (DGASA) et la Direction générale du personnel réseau et ministériel (DGPRM) du ministère de la Santé et des Services sociaux. Repéré à http://www.msss.gouv.qc.ca/ministere/acces_info/documents/demandes_acces/2016-2017/2 0162017-073-Document.pdf

\section{Pratiques de représentation collective au travail}

Dans la prochaine section, nous décrivons les pratiques de représentation collective des travailleuses à l'emploi des trois types de prestataires privés intégrés aux réseaux locaux de services ou qui concernent, directement ou indirectement, les conditions d'exercice de leur travail, et nous identifions les acteurs qui les portent.

\section{Travailleuses des EESAD}

Les pratiques de représentation collective des travailleuses à l'emploi d'EESAD recensées durant la période étudiée étaient toutes réalisées par des syndicats accrédités. Nous avons répertorié quinze EESAD syndiquées sur les 101 existantes. L'ensemble d'entre elles dispensaient des services d'assistance à la personne, selon le contenu des conventions collectives. Le personnel de 10 EESAD est syndiqué auprès du Syndicat québécois des employées et employés de service, section locale 298 (SQEES), affilié à la Fédération des travailleurs et travailleuses du Québec (FTQ); celui de 3 ÉESAD l'est auprès de la Centrale des syndicats démocratiques (CSD); celui d'une EESAD l'est auprès du Syndicat des Métallos (aussi affilié à la FTQ), alors que la dernière l'est auprès de la Confédération 
des syndicats nationaux (CSN). Les pratiques de représentation collective étaient centrées sur la négociation des conventions collectives avec la direction de chaque EESAD et sur le suivi de l'application de la convention. Dans la période couverte par notre étude, nous n'avons pas identifié de grève impliquant des travailleuses d'EESAD.

Nous avons identifié trois types de pratiques de représentation collective par des organisations syndicales à l'échelle nationale. La première s'inscrit dans le cadre de la participation de la CSN et de la FTQ au conseil d'administration du Chantier de l'économie sociale, un organisme non gouvernemental de promotion de l'économie sociale, créé à la suite du Sommet sur l'économie et l'emploi tenu en octobre 1996 à I'initiative de Lucien Bouchard, alors premier ministre du Québec. Avec ce Sommet consultatif, le gouvernement du Parti Québécois (PQ) visait à établir un partenariat social (Raymonde, 2013) axé sur le développement de la compétitivité économique, I'élimination du déficit et la redéfinition du rôle de l'État (Gouvernement du Québec, 1996: 7). Lors de ce Sommet, les directions des centrales syndicales ont manifesté leur soutien conditionnel au développement de l'économie sociale comme stratégie pour l'emploi dans le cadre d'une entente orale appelée "Consensus de 1996 " "11, entente qui impliquait aussi des représentants gouvernementaux, patronaux, communautaires et des groupes promoteurs de l'économie sociale. Cette entente stipulait que le panier de services d'aide à domicile offerts par les EESAD devait uniquement comporter des services d'aide domestique afin d'éviter la substitution des services publics d'assistance à la personne. Ce «Consensus» n'a cependant pas été défendu par l'ensemble des EESAD et de leurs regroupements (Boivin, 2013a) ${ }^{12}$ ni ne sera respecté par les autorités publiques lors de la réforme des services de 2003 (voir p. 508-509 de cet article). En 2013, les centrales syndicales, dans le cadre de leurs prises de position individuelles lors d'audiences publiques sur une proposition de réforme des services d'aide à domicile, ont continué à défendre le "Consensus de $1996 »($ CSN, 2013; FTQ, 2013). Cependant, durant les mêmes audiences, le Chantier de l'économie sociale (au sein duquel participent la CSN et la FTQ à travers leur représentation au conseil d'administration) s'est exprimé en accord avec la prestation de services d'assistance à la personne par les EESAD à certaines conditions (Chantier, 2013). Ces dernières sont, notamment, que les EESAD soient les seuls prestataires privés reconnus par l'État pour ces services, et que les salaires, les conditions de travail et la formation professionnelle du personnel s'améliorent par l'entremise d'un soutien adéquat de l'État.

La seconde pratique de représentation collective que nous avons identifiée durant la période étudiée est la participation de la CSN et de la FTQ à une campagne publique menée par les regroupements des EESAD et le Chantier de l'économie sociale pour une bonification du programme gouvernemental de 
financement des services d'aide domestique dispensés par les EESAD (Aile rurale et al., 2012), soit le Programme d'exonération financière pour les services d'aide domestique (PEFSAD). Ce programme, créé en 1997, ne finance pas les services d'assistance personnelle dispensés par les EESAD; ces derniers étant financés dans le cadre des contrats de services avec les CSSS.

Enfin, le troisième type de pratiques de représentation collective identifiée au niveau national est la participation de la CSN au Comité sectoriel de main d'œuvre en économie sociale et action communautaire (CSMO-ESAC), ainsi que celle de la Centrale des syndicats du Québec (CSQ), centrale syndicale très présente dans le secteur de l'enseignement. Le CSMO-ESAC, qui existe depuis 1997, figure parmi les 29 comités sectoriels de main-d'œuvre du Québec créés en vertu de la Loi favorisant le développement et la reconnaissance des compétences de la main-d'œuvre. Ces comités ont pour mission d'identifier les principaux problèmes de main-d'œuvre dans leur secteur d'activités et d'élaborer des actions pour y répondre ${ }^{13}$. En 2009, le CSMO-ESAC a contribué au développement d'un programme d'apprentissage en milieu de travail qui mène à l'octroi d'un "Certificat ministériel de qualification professionnelle» pour le métier de préposée d'aide à domicile, dont les tâches comportent exclusivement de l'aide domestique (Binhas, 2014). D'autres données indiquent qu'au cours de 2015-2016 14 le CSMO-ESAC a participé au développement d'une formation qui, cette fois, portait sur les services d'assistance à la personne. Elle s'adressera, à terme, à 4500 travailleuses des EESAD (CPMT, 2016) et a été financée dans le cadre d'un Plan d'action gouvernemental en économie sociale (Gouvernement du Québec, 2015). Cette formation est d'une durée de 120 heures (Lévesque, 2016), alors que la formation conduisant à un diplôme d'assistance à la personne à domicile exigée pour l'embauche des auxiliaires familiales et sociales dans le secteur public comporte 975 heures.

\section{Travailleuses du CES}

Nous n'avons recensé aucune accréditation syndicale comprenant une ou des travailleuses du CES, ni aucune pratique de représentation collective de celles-ci portée par une organisation syndicale ou un autre type d'association. Des pratiques de représentation collective concernant directement les conditions d'exercice du travail des salariées du CES ont, cependant, été portées par une coalition ad hoc formée de cinq associations locales de défense des droits des personnes vivant avec des limitations fonctionnelles de cinq localités différentes. Ces personnes constituent la majorité des usagers recourant au CES pour leurs services, comme indiqué précédemment. Créée en 2004, la coalition ne s'est pas donné de nom comme tel, mais elle regroupe les associations suivantes: I'Alliance sherbrookoise pour l'autonomie à domicile (ASAD), le Comité d'action 
des personnes vivant des situations de handicap (CAPVISH) de Québec, Ex Aequo de Montréal, le Regroupement des organismes de promotion des personnes handicapées (ROPPH) de Laval et le Service de références pour les personnes utilisant le Chèque emploi-service (PUCES) de Lanaudière (ASAD et al., 2004). Chacune de ces associations locales joue un rôle d'intermédiaire entre les travailleuses du CES et les usagers des services dans le cadre de leurs fonctions, tels le recrutement et le suivi administratif. Les travailleuses du CES n'étaient, toutefois, pas représentées dans les instances décisionnelles de ce regroupement.

En 2004, des rencontres d'organisation de cette coalition se sont tenues à plusieurs reprises et ont mené à une campagne d'action et à la publication d'un mémoire adressé aux députés de l'Assemblée nationale, au MSSS, ainsi qu'aux Agences de la santé et des services sociaux de chaque région du Québec. Les revendications portaient sur l'amélioration de la qualité des services et de celle de l'emploi afin de contrer la pénurie de main-d'œuvre. Les moyens ciblés étaient I'amélioration des salaires et des conditions de travail, la protection des droits du travail et des droits relatifs à la sécurité sociale, ainsi que l'accès à la formation continue pour les travailleuses du CES. La coalition revendiquait également que les personnes handicapées disposent d'un réel choix entre les services publics et ceux du CES. Elle soulignait l'écart entre la situation d'emploi des travailleuses du CES et celle du personnel du secteur public, tout en précisant ne pas remettre en question les salaires et les conditions de travail dans le secteur public (ASAD et al., 2004: 16). La coalition a fait pression sur les décideurs aux niveaux local, régional et national comme le révèle cet extrait d'entrevue avec une personne représentant une association membre de la coalition:

En même temps, on faisait des représentations à nos agences [régionales de la santé et des services sociaux], à nos CLSC. On travaillait au niveau local, au niveau régional, et au niveau provincial. On faisait tout ça en même temps pour essayer de faire avancer les affaires là. Puis, au niveau local, le CLSC nous disait: "C'est l'agence qui nous donne I'argent". Puis, quand on allait voir l'agence, ils nous disaient: "C'est le CLSC qui décide du taux horaire, ce n'est pas nous autres, ce n'est pas de notre responsabilité". Ils se « pitchaient» [renvoyaient] la balle de même.

En plus de produire un mémoire et d'effectuer des représentations politiques, la coalition a diffusé une pétition, envoyé aux autorités politiques une lettre signée par 76 travailleuses du CES et tenu une conférence de presse lors de laquelle certaines de ces travailleuses ont témoigné. La coalition a, finalement, été entendue par le MSSS. À la suite de ces actions, des gains salariaux importants, quoique ponctuels, ont été obtenus. À partir de 2005, des augmentations salariales ont été versées dans certaines régions. Le financement du programme du CES s'est accru de 10 millions de dollars en 2007-2008 et 2008-2009. Les salaires horaires ont été augmentés à $11 \$$ dans les régions du Québec, sauf 
dans celle de Montréal où ils ont été augmentés à 11,74 \$(AQRIPH, 2009: 11). Tout au long de la campagne de la coalition, soit de 2004 jusqu'à 2008, les augmentations obtenues furent de $32,5 \%$ en moyenne pour les régions de Sherbrooke et de Québec, tandis qu'elles furent de 14,5\% dans la région de Montréal parce que cette dernière bénéficiait d'une avance salariale depuis 1998. Par la suite, ce type de campagne publique n'a pas été poursuivi par la coalition ni initié par aucun autre organisme dans le domaine.

\section{Travailleuses d'agences de location de personnel}

Nous n'avons recensé aucune accréditation syndicale comprenant des travailleuses employées par des agences de location de personnel intégrées aux réseaux locaux de services d'aide à domicile, ni aucune pratique de représentation collective portée par des organisations syndicales ou par d'autres types d'organismes concernant les conditions d'exercice de leur travail dans le cadre de ces réseaux locaux. Dans le cadre de publications antérieures (Boivin, 2013a, 2015 et 2016), nous avons déjà traité d'un cas de pratiques de représentation collective par des salariées syndiquées dispensant des services d'aide à domicile employées par deux agences de location de personnel au Québec. Cependant, nous n'avons pas retenu ce cas pour le présent article car les contrats de services dans lesquels ces agences sont impliquées n'ont pas été établis avec un CSSS, mais plutôt avec un Centre de réadaptation public desservant des personnes présentant une déficience intellectuelle et vivant dans des résidences collectives.

\section{Discussion des résultats}

Nous avons constaté l'existence de certaines pratiques de représentation collective portées par les organisations syndicales au sein des services publics d'aide à domicile externalisés dans le cadre des réseaux locaux de services québécois durant la période 2003-2013. Elles ne peuvent cependant être qualifiées de pratiques de représentation collective réticulaire. Rappelons que nous définissions celle-ci comme un type de représentation collective prenant en compte le pouvoir stratégique exercé par l'organisation-cerveau dans les réseaux et visant la construction d'une solidarité et d'un pouvoir collectif des travailleurs et des travailleurs dépassant les divisions instaurées entre les segments des réseaux. Dans le cadre de notre recherche, nous avons également pu identifier un nouvel acteur porteur de pratiques de représentation collective concernant les conditions d'exercice du travail dans les services d'aide à domicile externalisés qui a déployé une «capacité d'influencer directement l'orientation des relations industrielles» (Bellemare, 2000: 386), même si son action a été très circonscrite dans le temps. 
Deux types de pratiques de représentation collective portées par les organisations syndicales ont été constatées à l'égard du personnel des EESAD. Le premier type est la représentation collective du personnel d'une quinzaine d'EESAD par des syndicats locaux. Nous l'avons identifié à une logique de représentation collective légaliste au sein de l'entreprise intégrée et de relations de travail bipartites. Ces syndicats locaux confinent, en effet, leur action à la sphère de l'employeur juridiquement reconnu, soit I'EESAD, et la centrent sur la négociation collective et le suivi de l'application de la convention collective. Le second type de pratiques de représentation collective, porté par des centrales syndicales, s'est manifesté dans le cadre de leur participation au Chantier de l'économie sociale et au Comité sectoriel de main-d'œuvre (CSMO-ESAC) aux côtés de représentants de divers milieux (groupes promoteurs de l'économie sociale, organismes communautaires, instances gouvernementales, etc.) en vue de développer l'emploi dans les EESAD et d'améliorer la qualification du personnel. Nous avons identifié ces pratiques à une logique de représentation collective partenariale. Comme le souligne Pinard, le partenariat laisse «à l'écart I'ensemble des salariés et des citoyens directement concernés (...). II n'est pas une forme d'action politique émancipatrice, car celle-ci implique que tous soient égaux et poursuivent des finalités communes. La fausse égalité propre au partenariat est manifeste dans l'objectif soi-disant commun de l'emploi » (Pinard, 2008: 16). Ainsi les positions du Chantier de l'économie sociale et du CSMO-ESAC, au sein desquels les centrales syndicales sont présentes, sont-elles contradictoires avec les positions que les centrales expriment individuellement lorsqu'elles se positionnent contre la substitution des services publics par ceux des EESAD (dans la logique du «Consensus de $1996 »)$.

Nous avons aussi pu identifier d'autres pratiques de représentation collective relatives aux conditions d'exercice du travail dans les services d'aide à domicile qui ne furent pas portées par des organisations syndicales, mais plutôt par une coalition ad hoc d'associations locales de défense de droits des personnes vivant avec des limitations fonctionnelles. Leurs pratiques se sont inscrites dans la sphère du pouvoir stratégique exercé par les autorités publiques, la coalition interpellant ces dernières au sujet des conditions d'exercice du travail dans le cadre du CES. Cette coalition peut être identifiée comme un nouvel acteur en relations industrielles (Bellemare, 2000), à la fois à cause de la dimension instrumentale de l'action et celle des résultats de celle-ci (Legault et Bellemare, 2008). Bien que le degré de continuité de l'action de la coalition soit limité, les augmentations salariales ponctuelles obtenues par la coalition ont été significatives. Cependant, il faut souligner une limite importante de ces pratiques collectives, soit l'absence de représentation collective des travailleuses du CES dans l'organe décisionnel de la coalition. Une autre raison qui empêche de parler d'une représentation collective réticulaire dans ce cas tient au fait que l'action de la coalition s'est 
centrée sur une catégorie de travailleuses seulement, sans prendre en compte la dynamique globale de pouvoir et de segmentation traversant l'organisation en réseau.

Enfin, I'absence de pratiques de représentation collective des travailleuses d'agences de location de personnel ne constitue pas un résultat empirique étonnant. Elle peut s'expliquer par les difficultés de constituer un collectif stable dans une agence, sans compter les difficultés de syndicalisation sur le plan juridique (Bernier, Vallée et Jobin, 2003).

\section{Conclusion}

De l'analyse des résultats de ces trois études de cas se dégage le constat d'une absence de représentation collective réticulaire dans le secteur des services d'aide à domicile au Québec. Autrement dit, une absence de pratiques collectives qui prennent en compte l'exercice du pouvoir stratégique (Appay, 1997) par les autorités publiques, dont l'action n'est aucunement régulée par le droit des rapports collectifs, et qui cherchent à construire une solidarité large pour contrecarrer la mise en concurrence de la main-d'œuvre employée dans les divers segments des réseaux locaux (Altmann et Dei $\beta$, 1998). À défaut d'une telle représentation collective réticulaire, la capacité des travailleuses employées dans le réseau apparait faible pour contrer la précarisation de l'emploi et la dévalorisation sexuée de leur travail traditionnellement identifié à la sphère domestique, au dévouement et à la disponibilité permanente (Boivin, 2013a et b). Évidemment, la généralisation analytique (Yin, 2003: 37) de nos résultats ne s'applique qu'au secteur des services d'aide à domicile au Québec. Des études dans d'autres secteurs d'activité et d'autres juridictions seront nécessaires pour élargir sa portée.

Sur le plan des implications concrètes de notre recherche pour les acteurs sur le terrain, il nous semble intéressant de souligner que les seules pratiques de représentation collective interpellant les autorités publiques quant à leur responsabilité à l'égard des travailleuses employées par des prestataires privés n'ont pas été portées par des syndicats, mais plutôt par des associations regroupant des usagers des services. Ce nouvel acteur dans les relations de travail (Bellemare, 2000) du secteur des services d'aide à domicile a, également, été identifié dans des études en Ontario (Hickey, 2012) et aux États-Unis (Boris et Klein, 2006). Sa mobilisation, qui lie qualité de l'emploi et des services, ouvre sur un potentiel d'alliances salariées-usagers, possiblement avec les syndicats et/ou d'autres types d'organismes (dont des groupes féministes ou de défense des droits des personnes migrantes, par exemple) afin d'instituer de nouvelles formes de représentation collective et une régulation juridique du travail plus protectrice pour les travailleuses, comme ce fut le cas au Québec dans le secteur des garderies à but non lucratif, devenues les Centres de la petite enfance (Bellemare et al., 2004). 
Un autre implication concrète qui ressort de notre recherche est la nécessité d'identifier la forme que pourrait prendre l'institutionnalisation d'une représentation collective réticulaire dans les services d'aide à domicile au Québec. Les possibilités offertes par la Loi sur les décrets de convention collective, qui permet à l'État d'étendre l'application d'une convention collective à tout un secteur d'activités, pourraient être étudiées, avec le soutien de juristes, dans la perspective d'une réforme progressiste de ce régime atrophié au fil des années (Bernier et Fontaine, 2012). Mais pour parvenir à une institutionnalisation répondant aux besoins collectifs des travailleuses et des usagers des services d'aide à domicile, nous pensons que la représentation collective réticulaire devra d'abord être mise en pratique et s'appuyer sur une vision commune de ce que devraient être la place et l'organisation des soins auprès d'autrui dans l'économie et la société.

\section{Notes}

1 Nous définissons l'externalisation comme le recours, par une entreprise ou un organisme public ou associatif, à une entité externe pour la réalisation de certaines de ses activités. Cette externalisation peut être effectuée dans une même juridiction ou un même pays ou encore par-delà ces frontières. Précisons que notre article traite d'externalisation au sein d'une même juridiction.

2 Nous définissons cette représentation collective comme la défense collective des intérêts d'un groupe de personnes, qu'elles soient ou non membres d'un syndicat (GumbrellMcCormick et Hyman, 2013: 52). Elle doit, néanmoins, reposer sur l'adhésion formelle des personnes représentées, ainsi que l'existence d'instances et de procédures décisionnelles démocratiques.

3 L'organisation en réseau est définie, dans notre recherche, comme le regroupement de plusieurs entités juridiquement autonomes et formellement liées autour de la production de biens ou services.

4 Nous utilisons la notion de "secteur public » et de "services publics» dans un sens large, en incluant à la fois la fonction publique (relevant directement des ministères gouvernementaux) et les organismes parapublics, qui comprennent, notamment, les établissements de santé et de services sociaux, dont ceux qui dispensent une partie des services d'aide à domicile.

5 Nous employons la forme féminine pour référer au personnel dispensant les services d'aide à domicile, car il est majoritairement féminin.

6 Nous avons renommé ainsi cette catégorie analytique afin de pouvoir l'appliquer à la fois aux entreprises privées et aux organismes publics.

7 Nous utilisons ces deux dernières catégories analytiques en nous inspirant à la fois des travaux d'Appay (2005) et d'Altmann et Dei $\beta$ (1998). "L'orchestration» est la mise en œuvre de restructurations dans l'organisation des services et leur répartition entre les diverses entités composant les réseaux locaux. La "régulation supra-organisation » est l'exercice, par I'organisation-cerveau, d'un contrôle direct ou indirect sur les activités des autres entités composant le réseau.

8 Pour une genèse plus détaillée des transformations organisationnelles des services publics d'aide à domicile au Québec, voir Boivin (2013a: 169-181; 293-296; 388-391). 
9 Le titre "auxiliaire familiale et sociale» a été regroupé avec d'autres au sein du nouveau titre " auxiliaires aux services de santé et sociaux» en 2005. Cependant, dans ce texte, nous utilisons I'ancienne formulation, qui est encore couramment utilisée.

10 Pour une présentation des modifications au Code des professions permettant ces situations, voir Boivin (2013a: 178).

11 L'engagement oral que constitue le «Consensus de 1996 » a été documenté par Vaillancourt et Jetté (2009: 32-38) qui ont analysé les 800 pages de transcriptions des délibérations du Sommet sur l'économie et l'emploi de 1996.

12 Selon des données provenant d'une enquête gouvernementale réalisée en 2009, 32 \% des EESAD offrent des services d'assistance à la personne (MAMROT, 2012: 11). La situation était très contrastée selon les régions du Québec, car dans 10 des 19 régions aucune EESAD n'offre ce type de services. Pour une présentation des différentes prises de positions des regroupements d'EESAD, voir Boivin (2013a: 326-331), ainsi que Vaillancourt et Jetté (2009).

13 II s'agit de la mission des comités sectoriels publiée sur le site Web de la Commission des partenaires du marché du travail, I'organisme consultatif qui les reconnait officiellement. Leur composition est décrite comme suit: «lls regroupent des représentants des employeurs ou d'associations d'employeurs et des représentants des syndicats ou d'autres représentants de travailleurs du secteur. S'y joignent également des représentants sans droit de vote de ministères et d'organismes à vocation sectorielle. Généralement présidés par un partenaire patronal et un partenaire syndical, les comités sectoriels fonctionnent selon une approche consensuelle». Consulté à l'adresse suivante: https://www.cpmt.gouv.qc.ca/reseau-despartenaires/comites-sectoriels.asp.

14 Ces données se situent en dehors de la période sur laquelle porte notre étude (2003-2013), mais comme elles s'inscrivent en continuité avec la position du Chantier de l'économie sociale exprimée en 2013, nous avons cru important de les prendre en compte dans la présente analyse.

\section{Bibliographie}

Aile rurale, Coalition des EESAD, FCSDSQ, CES, CQCM, CSN, FTQ, AQRP, Réseau FADOQ. 2012. "Aide à domicile: Lettre ouverte au Premier ministre, ministres et députés», consultée à: http://www.economiesocialemauricie.ca/?module=directory\&action=getMod\&subMod=CO M\&pclass=1\&uid=3098.

Alliance québécoise des regroupements régionaux pour l'intégration des personnes handicapées (AQRIPH). 2009. Il est moins cinq pour le chèque emploi-service. Mémoire, Montréal: AQRIPH.

Altmann, Norbert et Manfred Deiß. 1998. «Productivity by Systemic Rationalization: Good Work, Bad Work, No Work ?», Economic and Industrial Democracy, 19 (1), p. 137-159.

Appay, Béatrice. 1997. «Précarisation sociale et restructurations productives», dans B. Appay et A. Thébaud-Mony (dir.), Précarisation sociale, travail et santé, Paris: IRESCO, INSERM-CNRS, p. 509-554.

Appay, Béatrice. 1998. "Economic Concentration and Externalisation of Labour», Economic and Industrial Democracy, 19 (1), p. 161-184.

Appay, Béatrice. 2005. La dictature du succès. Le paradoxe de l'autonomie contrôlée et de la précarisation, Paris: L'Harmattan. 
Appay, Béatrice. 2008. "Précarité, précarisation. Vers un nouveau paradigme? », G. de Terssac et C. Saint-Martin (dir.), La précarité, une relation entre travail, organisation et santé, Toulouse: Octares, p. 161-170.

ASAD, CAPVISH, Ex æquo, ROPPH et PUCES. 2004. Chèque emploi-service: qu'en est-il de la dignité des personnes? Mémoire, Montréal : ASAD.

Avril, Christelle. 2009. "Une mobilisation collective dans I'aide à domicile à la lumière des pratiques et des relations de travail», Politix, 2 (86), p. 97-118.

Baudry, Bernard. 2005. L'économie des relations interentreprises, Paris: La Découverte.

Bélanger, Paul R., Benoît Lévesque et Marc Plamondon. 1987. «Flexibilité du travail et demande sociale dans les CLSC. Une étude de la convention collective et de la programmation. Rapport de recherche présenté à la Commission Rochon», Québec: Commission d'enquête sur les services de santé et les services sociaux.

Bellemare, Guy. 2000. "End Users: Actors in the Industrial Relations System ?», British Journal of Industrial Relations, 38 (3), p. 383-405.

Bellemare, Guy, Anne Renée Gravel, Louise Briand et Alain Vallée. 2004. "Syndicalisme et mouvements sociaux: voie de renouvellement des théories du syndicalisme et de l'action syndicale efficace? Le cas des services de garde », Économie et solidarités, 36 (2), p. 192-218.

Bernier, Jean, Guylaine Vallée et Carol Jobin. 2003. Les besoins de protection sociale des personnes en situation de travail non traditionnelle. Rapport final du Comité d'experts chargé de se pencher sur les besoins de protection sociale des personnes vivant une situation de travail non traditionnelle, Québec: Ministère du Travail.

Bernier, Jean et Laurence Léa Fontaine. 2012. L'extension juridique des conventions collectives au Québec: bilan et conditions d'une relance, Québec: Cahiers de I'Alliance de recherche universités-communautés Innovations, emploi et travail.

Bernstein, Stéphanie, Urwana Coiquaud, Marie-Josée Dupuis, Laurence Léa Fontaine, Lucie Morissette, Esther Paquet et Guylaine Vallée. 2009. "Les transformations des relations d'emploi: une sécurité compromise ?», Regards sur le travail, 6 (1), p. 19-29.

Béroud, Sophie. 2013. "Une campagne de syndicalisation au féminin. Une expérience militante dans le secteur de l'aide à domicile», Travail, genre et sociétés, 2 (30), p. 111-128.

Binhas, Lynda. 2014. Évaluation de l'implantation du PAMT Préposé(e) d'aide à domicile, Comité sectoriel de main d'œuvre en économie sociale et action communautaire (CSMO-ESAC).

Boivin, Louise. 2013a. Régulation juridique du travail, pouvoir stratégique et précarisation des emplois dans les réseaux: trois études de cas sur les réseaux de services d'aide à domicile au Québec, thèse de doctorat. Montréal: École de relations industrielles, Université de Montréal.

Boivin, Louise. 2013b. "Réorganisation des services d'aide à domicile au Québec et droits syndicaux: De la qualification à la disponibilité permanente juste-à-temps», Nouvelles Questions Féministes, 32 (2), p. 44-56.

Boivin, Louise. 2015. "Obstacles à l'exercice des droits syndicaux dans l'organisation en réseau. Le cas des services d'aide à domicile au Québec», Travail, emploi, formation, 13, p. 66-83.

Boivin, Louise. 2016. "Externalisation des services publics et division raciale du travail: la mobilisation novatrice de travailleuses d'agences», Vivre ensemble, Webzine, 23 (80). Consulté à http://www.cjf.qc.ca/upload/ve_bulletins/3712_a_Art_Louise-Boivin_No80.pdf. 
Boltanski, Luc et Ève Chiapello.1999. Le nouvel esprit du capitalisme, Paris: Gallimard.

Boris, Eileen et Jennifer Klein. 2006. "Organizing Home Care: Low-Waged Workers in the Welfare State», Politics and Society, 34 (1), p. 81-108.

Bourque, Raymonde. 1991. Évolution de la profession des auxiliaires familiales et sociales: significations et enjeux. Mémoire présenté comme exigence partielle de la maîtrise en sociologie, Montréal: UQÀM.

Chantier de l'économie sociale (2013). Mémoire présenté par le Chantier de l'économie sociale dans le cadre des consultations particulières sur le Livre blanc sur la création d'une assurance autonomie, Commission de la santé et des services sociaux, octobre 2013.

Christopherson, Susan et Jennifer Clark. 2007. Remaking Regional Economies: Power, Labor, and Firm Strategies in the Knowledge Economy, Londres et New York: Psychology Press.

Commission des partenaires du marché du travail (CPMT). 2016. Sommaire des réalisations de 2015-2016 et des perspectives de 2016-2017, Direction du développement des compétences et de l'intervention sectorielle.

Confédération des syndicats nationaux (CSN). 2013. L'autonomie pour tous. Mémoire présenté à la Commission de la santé et des services sociaux dans le cadre des consultations sur le Livre blanc, octobre 2013.

Cranford, Cynthia J. 2005. «From Precarious Workers to Unionized Employees and Back Again? The Challenges of Organizing Personal-Care Workers in Ontario », dans C. J. Cranford, J. Fudge et E. Tucker, dir., Self-Employed Workers Organize: Law, Unions and Policy, Montréal: McGill-Queens University Press, p. 96-135.

Da Roit, Blanche et Barbara Le Bihan. 2010. «Similar and Yet So Different: Cash for Care in Six European Countries' Long Term Care Policies», Milbank Quarterly, 88 (3), p. 286-309.

Davidov, Guy. 2015. «Indirect Employment: Should Lead Companies be Liable? », Comparative Labor Law and Policy Journal, 37 (1), p. 5-36.

Fédération des travailleurs et des travailleuses du Québec (FTQ). 2013. Mémoire de la FTQ sur le Livre blanc sur la création d'une assurance autonomie présenté à la Commission parlementaire sur la santé et les services sociaux, novembre 2013.

Gouvernement du Québec. 1996. Un Québec de solidarité et de responsabilité, document préparatoire de la Conférence sur le devenir social et économique du Québec, mars 1996.

Gouvernement du Québec. 2015. L'économie sociale. Des valeurs qui nous enrichissent. Plan d'action gouvernementale en économie sociale 2015-2020, Québec: Gouvernement du Québec.

Gumbrell-McCormick, Rebecca et Richard Hyman. 2013. Trade Unions in Western Europe: Hard Times, Hard Choices, Oxford: Oxford University Press.

Hickey, Robert. 2012. "End-Users, Public Services, and Industrial Relations: The Restructuring of Social Services in Ontario », Relations industrielles/Industrial Relations, 67 (4), p. 590-611.

Lamoureux, Diane. 1998. "La panacée de l'économie sociale: un placebo pour les femmes?», dans L. Boivin et M. Fortier, (dir.), L'économie sociale. L'avenir d'une illusion, Montréal: Fides, p. 25-53.

Legault, Marie-Josée et Guy Bellemare. 2008. "Theoretical Issues with New Actors and Emergent Modes of Labour Regulation», Relations industrielles/Industrial Relations, 63 (4), p. 742-768. 
Lévesque, Paul. 2016. Les entreprises d'économie sociale en aide domestique, Fédération des coopératives de services à domicile et de santé du Québec (FCSDSQ).

Mariotti, Fabien. 2005. Qui gouverne l'entreprise en réseau? Paris: Presses de Sciences Po.

Meintel Deirdre, Sylvie Fortin et Marguerite Cognet. 2006. "On the Road and on their Own: Autonomy and Giving in Home Health Care in Quebec », Gender, Place and Culture , 13 (5), p. $563-580$.

Ministère des Affaires municipales, des Régions et de l'Occupation du territoire (MAMROT). 2012. Profil des entreprises d'économie sociale en aide domestique. Partie 1. Tableau synthèse, Québec: MAMROT.

Ministère de la Santé et des Services sociaux (MSSS). 2003. Chez-soi: le premier choix. Politique de soutien à domicile, Québec: Gouvernement du Québec.

MSSS. 2004. Chez soi: le premier choix. Précisions pour favoriser l'implantation de la politique de soutien à domicile, Québec: Gouvernement du Québec.

MSSS. 2010. Nomenclature des titres d'emploi, des libellés, des taux et des échelles de salaire du réseau de la santé et des services sociaux à partir du 1"r avril 2010, Québec: Gouvernement du Québec

MSSS. 2011a. "Question 262 - Programme d'allocation directe», L'étude des crédits 20112012. Réponses aux questions particulières, volet services sociaux, Québec: Commission de la santé et des services sociaux, Volume 2, p. 102-103.

MSSS. 2011b. "Montant investi pour la main-d'œuvre indépendante pour les années 20052006 à 2009-2010 par région et par sous-catégorie d'emploi », L'étude des crédits 20112012. Réponses aux questions particulières, volet santé, 1, Québec: Gouvernement du Québec, p. 195-207.

MSSS. 2012. Tableaux présentant le nombre d'auxiliaires familiales et sociales (2005-2006) et d'auxiliaires aux services de santé et sociaux (2005-2006 à 2010-2011), Québec: Direction générale du personnel réseau et ministériel (DGPRM).

Moati, Philippe. 2006. «Relation d'emploi et frontières de la firme à l'épreuve des nouveaux rapports de subordination », dans $\mathrm{H}$. Petit et $\mathrm{N}$. Thèvenot (dir.), Les nouvelles frontières $d u$ travail subordonné. Approche pluridisciplinaire, Paris: La Découverte, p. 174-188.

Pinard, Rolande. 2000. La révolution du travail, de l'artisan au manager, Rennes: Presses universitaires de Rennes.

Pinard, Rolande. 2008. «Une affaire de société», Relations, 724, p. 15-17.

Raymonde, Ghislaine. 2013. Le «partenariat social». Sommet socio-économique de 1996, syndicats et groupes populaires, Montréal: M Éditeur.

Vaillancourt, Yves et Christian Jetté (avec la collaboration de Philippe Leclerc). 2009. Les arrangements institutionnels entre l'État québécois et les entreprises d'économie sociale en aide domestique: une analyse sociopolitique de l'économie sociale dans les services de soutien à domicile, Montréal: Éditions Vie économique.

Vallée, Guylaine. 2005. "Quelle responsabilité sociale pour l'entreprise ? Le cas du Canada», dans P. Auvergnon (dir.), Quelle responsabilité sociale pour l'entreprise? Approches juridiques nationales et comparatives, Bordeaux: Comptrasec, p. 59-94.

Weber, Florence, Loïc Trabut et Solène Billaud. 2014. Le salaire de la confiance. L'aide à domicile aujourd'hui. Paris: Rue d'Ulm Éditions. 
Weil David. 2014. The Fissured Workplace. Why Work Became so Bad for so Many and What Can be Done to Improve It. Cambridge: Harvard University Press.

Yin, Robert K. 2003. Case Study Research: Design and Methods, Thousand Oaks: Sage Publications.

Zeitlin, Jonathan. 2008. "Industrial Districts and Regional Clusters», dans G. Jones et J. Zeitlin (dir.), The Oxford Handbook of Business History, Oxford et Toronto: Oxford University Press, p. 219-243.

\section{RÉSUMÉ}

\section{La représentation collective au travail en contexte d'externalisation des services publics d'aide à domicile au Québec}

L'externalisation, qui donne lieu à l'intégration indirecte du travail dans l'organisation productive, pose des défis importants pour la représentation collective des travailleuses et des travailleurs. C'est que le droit du travail a été établi en fonction d'un tout autre modèle organisationnel. Afin de mieux comprendre ces défis, nous avons mené trois études de cas sur la représentation collective en contexte d'externalisation des services publics d'aide à domicile au Québec durant la période 2003-2013. Les travailleuses concernées - majoritairement des femmes — occupent des emplois précaires chez trois types de prestataires privés intégrés à des réseaux locaux de services: entreprises d'économie sociale en aide domestique (EE$S A D)$, usagers du programme Chèque emploi-service (CES) et agences de location de personnel. Nous avons examiné si des pratiques de représentation collective de ces travailleuses existent et quels acteurs sociaux les portent. Nous avons aussi vérifié si ces pratiques se confinent à l'intérieur des frontières de l'entité identifiée comme l'employeur au sens juridique ou si elles sont "réticulaires», étendant la solidarité à la sphère du pouvoir stratégique (Appay, 1997) exercé par les autorités publiques dans les réseaux.

Nos résultats montrent l'absence d'une représentation collective réticulaire dans ces réseaux locaux de services où la dévalorisation sexuée du travail, contrée en partie dans le secteur public, revient en force. Le personnel de 15\% des EESAD est syndiqué, mais les pratiques de représentation syndicale n'interpellent que l'employeur reconnu au sens juridique, les EESAD. Dans les agences de location de personnel intégrées à ces réseaux locaux, aucune forme de représentation collective n'existe, ni dans le programme CES. Cependant, une action collective interpellant les autorités publiques au sujet des conditions d'emploi dans le CES a eu un certain succès ponctuel. Portée par une coalition d'associations locales représentant des personnes vivant avec des limitations fonctionnelles, elle ouvre la voie à l'idée d'alliances salariées-usagers autour de la qualité des services et de l'emploi.

MOTS-CLÉs: réseau, syndicalisme, relations de travail triangulaires, précarité, privatisation. 


\section{SUMMARY}

\section{Collective Representation at Work in the Context of Outsourcing of Public Homecare Services in Quebec}

Outsourcing, which entails the indirect integration of work into the organization of production and services, poses significant challenges for workers' collective representation. This is because the relevant labour law is premised on a completely different organizational model. To gain insight into these challenges, three case studies were conducted on collective representation in the context of outsourcing of public homecare services in Quebec during the 2003-2013 period. The workers involved-for the most part women-held precarious jobs in three types of private service providers integrated into local networks of services: social economy domestic help enterprises (EESAD), users of the Service Employment Paycheque plan (SEP) and employment agencies. We examined whether these workers are represented collectively and which social actors are involved. We also investigated whether these practices are confined within the boundaries of the entity identified as the employer in the legal sense or whether they are "reticular", extending solidarity to the sphere of strategic power (Appay, 1997) exercised by the public authorities in the networks.

Our results show that there is no reticular collective representation in these local networks of services where the gendered devaluation of work, partly overcome in the public sector, is back with a vengeance. Employees in $15 \%$ of EESADs are unionized but the union representation practices only focus on the legally recognized employer, the EESADs. In the employment agencies integrated into these local networks, no form of collective representation exists, nor does such representation exist in the case of the SEP. However, collective action targeting the public authorities involving working conditions under the SEP has had some oneoff success. Driven by a coalition of local associations representing people living with disabilities, it paves the way for the idea of employee-user alliances around service and job quality.

KEYWORDS: network, trade unionism, triangular employment relationships, precariousness, privatization.

\section{RESUMEN}

\section{La representación colectiva laboral en contexto} de externalización de servicios públicos de asistencia a domicilio en Quebec

La externalización, que da lugar a la integración indirecta del trabajo en la organización productiva, plantea retos importantes a la representación colectiva de los trabajadores y trabajadoras. Es que el derecho laboral ha sido establecido en función de un modelo organizacional diferente. Para comprender mejor estos retos, 
hemos llevado a cabo tres estudios de caso sobre la representación colectiva en contexto de externalización de servicios públicos de asistencia domestica a domicilio en Quebec durante el periodo 2003-2013. Las trabajadoras concernidas-mayoritariamente mujeres-ocupan empleos precarios en tres tipos de proveedores privados integrados a las redes locales de servicios: las empresas de economía social de asistencia domestica (EESAD), los utilizadores del programa Cheque empleoservicios (CES) y las agencias de empleo. Hemos examinado si las prácticas de representación colectiva de esas trabajadoras existen y cuáles son los actores sociales que los sostienen. Hemos verificado también si esas prácticas son confinadas al interior de las fronteras de la entidad identificada como empleador en términos jurídicos o si son "reticulares», extendiendo la solidaridad a la esfera del poder estratégico (Appay, 1997) ejercido por las autoridades públicas en las redes.

Nuestros resultados muestran la ausencia de una representación colectiva reticular en esas redes locales de servicios donde la desvalorización sexuada del trabajo, bloqueada en parte en el sector público, regresa con fuerza. El personal de $15 \%$ de las EESAD es sindicalizado pero las prácticas de representación sindical interpelan únicamente al empleador jurídicamente reconocido, las EESAD. En las agencias de empleo integradas a esas redes locales, no existe ninguna forma de representación colectiva, ni en el programa CES. Sin embargo, una acción colectiva interpelando las autoridades públicas en cuanto a las condiciones de empleo en las CES tuvo cierto éxito puntual. Sostenida por una coalición de asociaciones locales que representaban las personas que viven con limitaciones funcionales, la acción abrió la vía a la idea de alianzas entre asalariados y utilizadores de servicios en torno a la calidad de los servicios y del empleo.

PALABRAS CLAVES: red, sindicalismo, relaciones de trabajo triangulares, precariedad, privatización. 